\title{
Construção de um Instrumento para Avaliação de Satisfação da Atenção Básica nos Centros Municipais de Saúde do Rio de Janeiro
}

\section{Construction of a Tool for Evaluating Patient Satisfaction in Primary Care Units of Rio de Janeiro}

Vera Lucia Rabello Castro Halfoun ${ }^{I}$ Odaleia Barbosa de Aguiar ${ }^{I L}$ Denise da Silva Mattos ${ }^{I}$

PALAVRAS-CHAVE:

- Atenção primária;

- Satisfação;

- Qualidade de atendimento.

KEY WORDS:

- Primary care;

- Satisfaction;

- Quality of care services.

Recebido em: 20/04/2006

Reencaminhado em: 09/07/2007

Aprovado em: 03/01/2008
${ }^{I}$ Universidade Federal do Rio de Janeiro, Rio de Janeiro, Brasil.

"Universidade do Estado do Rio de Janeiro, Rio de Janeiro. Rio de Janeiro, Brasil. 


\section{INTRODUÇÃO}

Em 1999, a Faculdade de Medicina iniciou um projeto de atendimento à população de pacientes hipertensos e de diabéticos, no âmbito dos programas específicos de um Centro Municipal de Saúde (CMS) localizado no Rio de Janeiro ${ }^{1,2}$. A literatura em geral mostra como, apesar de todos os avanços diagnósticos e terapêuticos, ainda é bastante insatisfatório o controle de enfermos com doenças crônico-degenerativas que cursam assintomáticas por longo tempo em sua história natu$\mathrm{ral}^{3,4}$. A baixa aderência ao tratamento e a alta taxa de abandono à consulta impõem um desafio aos profissionais de saúde, no sentido de buscar estratégias que possam suplantar estas dificuldades. O modelo de assistência centrado no médico não tem sido capaz de superar este desafio.

Nesse projeto, o modelo alternativo proposto tem como princípio o atendimento interdisciplinar, com abordagem integral no acompanhamento de hipertensos e/ou diabéticos com foco no controle da(s) doença(s), aumento da aderência ao tratamento e redução do abandono às consultas por meio da melhora da satisfação dos usuários e de sua qualidade de vida e da redução dos custos em relação ao atendimento tradicio$\mathrm{nal}^{5}$. A construção do projeto em equipe permitiu uma reflexão sobre a influência da relação profissional-paciente, a organização do atendimento nas diferentes clínicas e a integração dos profissionais na busca de um resultado satisfatório.

Entendendo que a qualidade do atendimento é uma variável fundamental para garantir a satisfação do usuário, a equipe responsável pelo projeto considerou que um questionário deveria abordar as condições de acessibilidade, satisfação com serviços disponíveis, infra-estrutura, além da satisfação geral com o atendimento da unidade. Desta forma, a variável abstrata que se pretendeu medir foi a satisfação do usuário, expressa pela qualidade da atenção ${ }^{6}$.

A elaboração de um instrumento de medida de variáveis que sejam observáveis e diretamente relacionadas ao objeto de estudo deve ser adequada à população a ser investigada, ou seja, o instrumento deve ser compreendido da mesma forma por todos os que responderão ao questionário. Sua construção deve, portanto, ser feita com a participação de diferentes populações que se relacionem de alguma forma com o objeto da avaliação ${ }^{6}$.

Instrumentos de medida costumam geram pontuações objetivas que denotam a magnitude do evento que queremos medir. Normalmente, tentamos avaliar esta magnitude e se há discrepâncias entre o que o instrumento de medida é capaz de medir e a magnitude real da variável, ou seja, a confiabilidade do instrumento ${ }^{6}$. A confiabilidadeindica a extensão na qual mensurações obtidas sob diferentes circunstâncias proporcio- nam resultados semelhantes. $\mathrm{O}$ coeficiente de confiabilidade reflete a extensão em que um instrumento de mensuração consegue discriminar os indivíduos, refletindo as verdadeiras diferenças entre eles ${ }^{7}$.

A validade pode ser expressa pela pergunta: o instrumento mede efetivamente o que desejamos medir? A validade é comumente avaliada quanto a conteúdo, critério e constructo. A validade de conteúdo tem por objetivo verificar se as perguntas são a expressão observável do constructo e é feita por especialistas que julgam até que ponto o instrumento é representativo do que se quer medir. A validação de critério estabelece as relações existentes entre as pontuações exibidas pelo instrumento e aquelas obtidas por um outro critério que defina, de forma adequada e independente, o que se quer medir (padrão ouro). A busca de um critério ideal nem sempre é fácil e, na prática, pode ser obtida pela comparação do instrumento recém-criado com outro já existente e que tenha razoável aceitação e consenso. A validade do constructo é usualmente buscada quando não se podem inferir informações adequadamente por meio de um critério ou de um conteúdo. Quando não houver padrão ouro para se comparar, estudos repetidos que convergem na mesma direção devem ser realizados como um processo contínuo ${ }^{6}$.

O objetivo do presente estudo foi construir e validar um questionário para avaliar a satisfação dos usuários de uma unidade básica do Rio de Janeiro que possa representar um instrumento de avaliação do projeto de hipertensão arterial desenvolvido pela Faculdade de Medicina e que possa também ser utilizado pela rede básica de CMS.

\section{METODOLOGIA}

No segundo semestre de 2000, no âmbito do projeto de hipertensão arterial e de diabetes mellitus, numa primeira etapa, 15 profissionais da UFRJ com exercício no CMS e oito alunos no segundo período do curso médico foram divididos em quatro grupos para elaboração de questões classificadas em: (a) acesso ao CMS; (b) consulta; (c) pós-consulta; (d) outras atividades complementares. Resultaram 96 questões, sendo uma mais geral em relação ao atendimento (Quão satisfeito você está com o atendimento do CMS em geral?) e outras específicas sobre os seguintes temas e subtemas:

(a) acesso - facilidade de acesso, sinalização, informações sobre o CMS, número de tentativas para abrir prontuário e para obter consulta, facilidade de circulação e orientação dentro do CMS, acomodações e infra-estrutura do CMS, consulta com hora marcada, horário de funcionamento do CMS, tempo de espera, conforto de instalações; 
(b) consulta - satisfação com o profissional, compreensão sobre a doença, compreensão sobre a indicação de exames, compreensão sobre significação dos resultados de exames, informação sobre a realização dos exames, compreensão sobre o tratamento dietético e efeitos colaterais, compreensão sobre tratamento medicamentoso, informação sobre efeitos colaterais dos medicamentos, compreensão sobre cuidados gerais e expectativa de melhora com a dieta, com a consulta e com a medicação;

(c) pós-consulta - recebimento da medicação, orientação sobre a medicação durante a dispensação e realização dos exames, reclamações e sugestões;

(d) outros - atividades em grupos educativos e de dança.

Foram realizadas reuniões com toda a equipe (quatro médicos, cinco enfermeiros, quatro nutricionistas, dois técnicos de enfermagem e oito alunos), seguidas de reuniões com oito pacientes usuários do sistema de saúde, seis pessoas não usuárias do CMS, três profissionais da área administrativa e novamente com toda a equipe, perfazendo um total de seis reuniões, para simplificar o conteúdo, evitar repetições e melhorar o entendimento das questões, avaliando, portanto, a adequação do questionário à população estudada. Deste processo resultaram 46 questões. As três questões sobre grupos educativos e sobre a participação no grupo de dança não foram utilizadas no presente estudo, pois os pacientes que participaram do processo não participaram destas atividades. Numa etapa seguinte, as questões foram analisadas por especialistas da atenção básica, composta por três clínicos, dois pediatras, um sanitarista, duas enfermeiras com formação em saúde pública e dois gestores em saúde, em reunião específica, para verificar a validade de conteúdo do questionário.

A versão final do questionário foi composta de: quatro questões com respostas por escalas de satisfação que variaram de extremamente satisfeito, bastante satisfeito, mais ou menos satisfeito, pouco satisfeito e nada satisfeito. Em alguns itens, como uso de medicação, realização de exercícios físicos programados pelo CMS, prescrição de dieta e outros, compreendendo 30 questões, as respostas foram sim ou não. Em caso de resposta negativa, as questões subseqüentes referentes ao respectivo item eram puladas. Desta forma, como exemplo, quem respondia que não recebeu prescrição medicamentosa não era inquirido sobre a compreensão da ação do medicamento ou seus efeitos colaterais. Três questões foram organizadas com respostas em números de 1 a 5 (tentativas de abrir prontuários ou obter a primeira consulta e horas de espera para atendimento). Finalmente, foram organizadas seis questões que previam uma resposta entre várias opções propostas.
A versão final obtida do processo anterior foi aprovada para aplicação em duas etapas de pré-testes. O objetivo de cada etapa foi aperfeiçoar o conjunto de itens e as escalas que englobaram os quatro blocos temáticos com as 43 perguntas.

O primeiro pré-teste (23 voluntários) foi respondido por pacientes que se encontravam nas salas de espera de um hospital universitário do Rio de Janeiro, que respondiam o questionário para o entrevistador. Avaliava-se a clareza da formulação das perguntas e verificavam-se as dificuldades encontradas em respondê-las. As alterações sugeridas foram incorporadas.

O segundo pré-teste foi realizado com 20 voluntários oriundos de cinco unidades municipais de atenção básica (primária) que responderam as questões reformuladas.

Um estudo piloto foi realizado com 47 pacientes de um CMS que responderam ao mesmo questionário (reteste) após sete dias.

Para aplicação do questionário, os alunos foram treinados para abordagem e relacionamento com os pacientes selecionados entre aqueles que esperavam a consulta e concordaram em respondê-lo, e nas técnicas de leitura, em seminário específico para realizar o estudo piloto.

A confiabilidade foi testada calculando-se a concordância entre as respostas às questões nos dois momentos e os valores de consistência interna pelo coeficiente alfa de Cronbach e pelo coeficiente de Kuder-Richardson fórmula - 20 (KR-20).

Um termo de consentimento escrito, livre e esclarecido foi assinado pelos pacientes. Os analfabetos, após consentimento verbal, tiveram seus formulários assinados por duas testemunhas não envolvidas no estudo. O protocolo foi aprovado pela Comissão de Ética do Centro de Ciências da Saúde da UFRJ, localizado no Hospital Universitário Clementino Fraga Filho/ UFRJ, sob o número 36/99 em 06/05/99.

\section{RESULTADOS}

As questões propostas de início foram progressivamente melhoradas em relação à clareza e objetividade, sendo reduzidas a 43 no formato final do questionário, sendo a primeira de caráter geral (De maneira geral, quão satisfeito você está com o atendimento deste Centro Municipal de Saúde (CMS)?). As questões sobre tempo de espera para consulta, número de tentativas para obter marcação de consulta, facilidades de acesso ao CMS e de deslocamento interno nas dependências do CMS, facilidades de informação sobre serviços, conforto de instalações, recebimento de dieta, medicação e facilidade de realização de exames foram consideradas indicadores de qualidade da unidade básica. Aquelas referentes à compreensão sobre indicação e resultado de exames e ao tratamento e seus riscos foram indicadoras da qualidade do atendimento presta- 
do pelos profissionais. De modo geral, foram organizadas em ordem, de acordo com as etapas percorridas pelos pacientes desde sua entrada no CMS até a saída.

O questionário foi considerado apto para ser aplicado após a obtenção de consenso entre os especialistas especial- mente convidados a opinar sobre seu conteúdo e a análise de confiabilidade das respostas dos 47 pacientes testados por duas vezes, com intervalo de uma semana, sendo o percentual de concordância maior do que $90 \%$ para a maioria das questões (Tabelas de 1 a 4 ).

TABELA 1

Questões com tipo de respostas sim ou não

\begin{tabular}{|c|c|c|}
\hline Temáticas & Questões & \% concordância \\
\hline \multirow[t]{2}{*}{ Sinalização no CMS } & As informações sobre as dependências do posto estão apresentadas de forma clara & 97,87 \\
\hline & O deslocamento nas dependências internas do posto é fácil & 93,61 \\
\hline Informação sobre os serviços & As informações sobre os serviços oferecidos pelo CMS estão apresentadas de forma clara & 100 \\
\hline \multirow[t]{2}{*}{ Conforto nas instalações } & Estou satisfeito com as dependências do CMS & 100 \\
\hline & O CMS tem acomodação para todos enquanto aguardam o atendimento & 97,87 \\
\hline \multirow[t]{3}{*}{ Qualidade do profissional } & Eu me senti seguro em relação ao profissional que me atendeu & 100 \\
\hline & Eu fiquei satisfeito com o exame físico realizado durante a consulta & 97,87 \\
\hline & Eu fiquei satisfeito com a entrevista feita durante a consulta & 95,74 \\
\hline Compreensão sobre a doença & Eu compreendi os problemas que esta doença pode causar & 97,87 \\
\hline \multirow[t]{9}{*}{ Informação sobre exames* } & Nos últimos dois meses foi solicitado algum exame para você? & 95,74 \\
\hline & Eu compreendi a necessidade de realizar os exames & 100 \\
\hline & Os exames realizados me causaram desconforto & 87,50 \\
\hline & Eu estava ciente do desconforto causado pelo exame & 100 \\
\hline & Eu pude me submeter aos exames solicitados & 100 \\
\hline & Eu realizei os exames solicitados & 100 \\
\hline & Me recusei a fazer os exames solicitados & 0 \\
\hline & Eu soube do resultado dos exames & 90,62 \\
\hline & O resultado dos exames mudou minha conduta em relação à doença & 100 \\
\hline \multirow[t]{4}{*}{ Compreensão sobre a dieta } & Você recebeu prescrição de alguma dieta? & 72,34 \\
\hline & Eu compreendi a importância da dieta para o tratamento de minha doença & 100 \\
\hline & Eu compreendi por que fazer a dieta no horário e da maneira receitada & 95 \\
\hline & Eu compreendi muito bem como fazer a dieta & 83,33 \\
\hline \multirow[t]{5}{*}{ Compreensão sobre a medicação } & Você recebeu prescrição de algum medicamento? & 97,87 \\
\hline & Eu compreendi a importância da medicação para o tratamento de minha doença & 97,72 \\
\hline & Eu compreendi por que tomar a medicação no horário e da maneira receitada & 97,72 \\
\hline & Eu compreendi os problemas que a medicação pode causar & 100 \\
\hline & Minhas dúvidas sobre a medicação foram esclarecidas & 95,45 \\
\hline Reclamações e sugestões & O CMS oferece condições para receber e atender reclamações e sugestões? & 100 \\
\hline
\end{tabular}

* Esta questão foi agrupada em uma única questão, com opções de resposta sim e não para cada item.

TABELA 2

Questões com respostas graduadas por graus de satisfação

\begin{tabular}{|c|c|c|}
\hline Temáticas & Questões & \% concordância \\
\hline Acesso da residência ao CMS & O quão satisfeito você está com o acesso da sua casa a este serviço de saúde? & 95,74 \\
\hline Consulta com hora marcada & O quão satisfeito você ficaria se o seu atendimento fosse com hora marcada? & 93,62 \\
\hline Tempo de espera de consulta & $\begin{array}{l}\text { O quão satisfeito você está com o tempo de espera entre a hora de sua chegada ao CMS e a } \\
\text { hora em que você foi consultado ou teve outra atividade neste serviço? }\end{array}$ & 95,74 \\
\hline Atendimento do CMS & O quão satisfeito você está com o atendimento deste CMS Marcolino Candau? & 97,29 \\
\hline
\end{tabular}

Os graus de satisfação foram escalonados em extremamente, bastante, mais ou menos, pouco ou muito pouco satisfeitos. 
TABELA 3

Questões com respostas quantificadas por graus numéricos

\begin{tabular}{ll}
\hline \multicolumn{1}{c}{ Temáticas } & \multicolumn{1}{c}{ Questões } \\
\hline Obtenção da $1^{\text {a }}$ consulta & Quantas vezes você precisou vir ao CMS para ter seu primeiro atendimento efetivado? \\
Abertura do prontuário & Quantas vezes você precisou vir ao CMS para abrir prontuário? \\
Tempo de espera & Quantas horas, na maioria das vezes, você aguarda na sala de espera para ser atendido? \\
\hline
\end{tabular}

A quantificação foi feita para 1, 2, 3, 4 e 5 ou mais.

TABELA 4

Questões redigidas para escolha de uma opção de resposta

\begin{tabular}{|c|c|c|}
\hline Temáticas & Questões & \% concordância \\
\hline $\begin{array}{l}\text { Significação dos resultados } \\
\text { de exames }\end{array}$ & $\begin{array}{l}\text { Eu entendi perfeitamente o significado dos resultados dos exames } \\
\text { Eu tive dúvidas sobre o significado dos resultados dos exames } \\
\text { Eu não entendi o significado do resultado dos exames }\end{array}$ & 87,5 \\
\hline $\begin{array}{l}\text { Informação sobre realização } \\
\text { dos exames }\end{array}$ & $\begin{array}{l}\text { O exame foi marcado para antes da consulta agendada } \\
\text { O exame foi marcado para depois da consulta agendada } \\
\text { Não consegui marcar o exame }\end{array}$ & 100 \\
\hline $\begin{array}{l}\text { Compreensão sobre o } \\
\text { tratamento dietético }\end{array}$ & $\begin{array}{l}\text { Minhas dúvidas sobre a dieta foram esclarecidas } \\
\text { Parte de minhas dúvidas sobre a dieta foi esclarecida } \\
\text { Minhas dúvidas sobre a dieta não foram esclarecidas }\end{array}$ & 90 \\
\hline $\begin{array}{l}\text { Expectativa de melhora } \\
\text { com a dieta }\end{array}$ & $\begin{array}{l}\text { Eu vou melhorar se fizer a dieta } \\
\text { A dieta não tem influência sobre meu problema de saúde } \\
\text { Eu vou piorar com a dieta }\end{array}$ & 95 \\
\hline Recebimento da medicação & $\begin{array}{l}\text { Recebi no posto toda a medicação que me foi prescrita } \\
\text { Recebi no posto parte da medicação que me foi prescrita } \\
\text { Não recebi do posto toda a medicação que me foi prescrita }\end{array}$ & 95,45 \\
\hline $\begin{array}{l}\text { Expectativa de melhora } \\
\text { com a consulta }\end{array}$ & $\begin{array}{l}\text { Eu me senti melhor logo após a consulta } \\
\text { Eu me senti confiante em melhorar após o tratamento } \\
\text { Aguardarei o resultado do tratamento para avaliar se vou melhorar } \\
\text { Continuei inseguro em relação às possibilidades de melhora } \\
\text { Não acredito que vou melhorar }\end{array}$ & 95,74 \\
\hline
\end{tabular}

A Tabela 5 apresenta os valores da consistência interna (coeficiente alfa de Cronbach) e de Kuder-Richardson fórmula - 20 (KR-20). Para cálculo destes coeficientes, as questões foram agrupadas segundo as temáticas: acesso, satisfação com o atendimento, compreensão e uso de medicação, solicitação de exames e infra-estrutura do serviço. Os valores foram superiores a 0,80 , ou seja, mostraram boa consistência interna para as questões sobre acesso, satisfação com o atendimento e infra-estrutura; as questões referentes ao uso da medicação e à solicitação de exames mostraram valores um pouco menores.

\section{TABELA 5}

Avaliação da consistência interna: coeficientes utilizados

\begin{tabular}{llc}
\hline \multicolumn{1}{c}{ Coeficientes } & \multicolumn{1}{c}{ Dimensões } & Total \\
\hline alfa de Cronbach & acesso & 0,87 \\
& satisfação com atendimento & 0,88 \\
Kuder-Richardson & compreensão e uso de medicação & 0,77 \\
fórmula-20 (KR-20) & solicitação de exame & 0,76 \\
& acesso: infra-estrutura & 0,94 \\
\hline
\end{tabular}

\section{DISCUSSÃO}

A opção de construir um novo questionário como estratégia mais adequada para o estudo de satisfação dos usuários de atenção primária foi justificada pelas peculiaridades de funcionamento dos centros municipais de Saúde do Rio de Janeiro em relação às outras formas de organização da atenção básica e de outros níveis de atenção. Desta forma, a estruturação do questionário seria importante como modelo para aplicação em outros centros municipais no município do Rio de Janeiro.

Tendo em vista que o princípio básico sobre o qual as questões foram formuladas foi a satisfação do usuário expressa na qualidade do atendimento, algumas das questões focalizam a prática do atendimento à saúde. Assim, por exemplo, o questionário pode avaliar quantos pacientes receberam orientação dietética, solicitação de exames, prescrição medicamentosa, ou quantos compreenderam o significado de sua doença, os efeitos de seus remédios ou ainda o que significa o resultado de um exame, indicadores de qualidade da consulta realizada. 
A taxonomia dos temas específicos de questões foi obtida na fase inicial de construção, com base nas especificidades de serviços do CMS e no foco de interesse da equipe. Desta forma, os cinco primeiros grupos foram divididos segundo as categorias acesso, consulta, pós-consulta e outras atividades, de acordo com o fluxo do deslocamento habitual do paciente no CMS. Howell e Concato aplicaram, em 63 gestantes de uma clínica obstétrica, perguntas abertas sobre satisfação com o atendimento e as organizaram taxonomicamente ${ }^{8}$. Pelas respostas das pacientes, as perguntas foram divididas em seis eixos principais, relacionados aos médicos, enfermeiras, outros funcionários, pessoal de métodos especiais, atributos do hospital e foco pessoal, num total de 51 itens pesquisados. Após a validação do questionário, os autores o consideraram simples e adequado à aplicação. No presente estudo o interesse foi dirigido à satisfação com os serviços prestados, independentemente do pessoal que os oferecia.

A metodologia utilizada no questionário atende aos prérequisitos de adequação, confiabilidade e validade recomendados $^{6}$. A adequação das questões foi observada pelo processo de discussão com populações diferentes, pessoal de saúde, pacientes usuários, não usuários do CMS e alunos, com o objetivo de avaliar a clareza, a definição e se todos compreenderam as perguntas da mesma forma.

Houve concordância superior a $90 \%$ entre as respostas dadas pelos mesmos pacientes em ocasiões diferentes para a maioria das questões, atestando a confiabilidade do questionário ${ }^{6}$. Apenas a questão referente ao recebimento de dieta pela unidade mostrou um percentual de concordância inferior a $80 \%$ (Tabela 1). A possibilidade de os pacientes terem recebido a dieta no intervalo entre as duas aplicações do questionário não pode ser excluída, o que justificaria as discordâncias apresentadas em quase 30\% dos pacientes. Da mesma forma, os valores dos coeficientes alfa de Cronbach e de Kuder-Richardson fórmula - 20 (KR-20) atestam a confiabilidade das questões, alta para a imensa maioria.

A validade de conteúdo se observou nas discussões feitas por especialistas no assunto, tendo em vista tratar-se de pessoal que trabalhava nesta área e que tinha experiência e titulação acadêmicas. Houve um consenso entre estes especialistas, obtido em reunião convocada para este fim.

A análise pelos coeficientes estudados mostrou boa consistência interna do instrumento, considerando-se como ponto de corte 0,8 . As dimensões solicitação de exames e compreensão e uso da medicação tiveram índices levemente inferiores, o que, no contexto geral do instrumento, não foi valorizado, não comprometendo a possibilidade de uso do questionário.

\section{CONCLUSÃO}

Um questionário específico para análise da satisfação de usuários, centrado na qualidade do atendimento, foi desenvolvido e validado para aplicação em centros municipais de Saúde do município do Rio de Janeiro.

\section{AGRADECIMENTOS}

Aos alunos do curso médico Leandro Albuquerque Lemgruber Kroft, Juliano Carvalho Gomes de Almeida, Taissa Sotto Mayor Xavier, Ricardo Carmo Bastos, Felipe Neves de Albuquerque, Flavia Porto Cardozo, Rafael Potter Soeiro Pinto, André Felipe Pereira Reiol Santos, pelo entusiasmo, interesse acadêmico e estímulo que proporcionaram a seus mestres e colegas no decorrer deste trabalho. À Fundação Universitária José Bonifácio, pelo apoio financeiro ao projeto.

\section{REFERÊNCIAS}

1. Ministério da Saúde, 1998. Normas técnicas para o programa nacional de educação e controle da hipertensão arterial. Brasília.

2. Ministério da Saúde. Secretaria nacional de programas especiais de saúde. Manual de diabetes. Divisão nacional de doenças crônico-degenerativas. Brasília; 1990.

3. Welch VL, Hill MN. Effective strategies for blood pressure control. Cardiol Clin 2002; 20 (2): 321-333.

4. Baltazar J, Natario A. [An evaluation of the quality of medical care for hypertensive patients. A review of the record of explicit criteria and standard in the clinics of District of Lisbon]. Acta Med Port 1993; 6 (10): 431-438.

5. Halfoun VLRC, Araujo MC, Malveira EAP, Gonçalves MC, Griep RH, Mattos D et al. Custo/efetividade de um modelo assistencial multidisciplinar nos programas de hipertensão arterial e diabetes mellitus em uma unidade de saúde do município do Rio de Janeiro. In: Anais do VI Congresso Brasileiro de Saúde Coletiva, Ciência e Saúde Coletiva; Salvador, Brasil. Rio de Janeiro: ABRASCO; 5 (supl): 193, 2000. (resumo).

6. Prieta L, Badia X. Cuestionarios de Salud: concepto e metodologia. Atencion Primaria 2001; 28(3): 201-209.

7. Streiner DL e Norman GR. Basic Concepts in Health measurement scales. A practical guide to their development and use. Oxford University Press Inc, New York. 1995.

8. Howell EA, Concato J. Obstetric patient satisfaction: Asking patients what they like. Am J Obstet Gynecol. 2004 Jan;190 (1):175-182.

9. Halfoun VLRC; Mattos, DS.; Aguiar, OB. A satisfação do usuário em dois modelos de atendimento no Programa de 
Hipertensão Arterial em uma unidade de saúde do município do Rio de Janeiro. Cadernos saúde coletiva. 2005; 13(3): 16-42.

\section{CONFLITOS DE INTERESSE}

Declarou não haver.

\section{ENDEREÇO PARA CORRESPONDÊNCIA}

Centro Municipal de Saúde Marcolino Candau Rua Laura de Araújo 36,

CEP 20211-170 Rio de Janeiro / RJ

E-mail: halfounv@uol.com.br 